\title{
The Concept of a System for Identification of the Dangerous Areas for People with Visual Dysfunction
}

\author{
J. WICIAK* AND B. BORKOWSKI \\ Department of Mechanics and Vibroacoustics, AGH - University of Science and Technology \\ al. A. Mickiewicza 30, 30-059 Kraków, Poland
}

\begin{abstract}
The article presents the concept of a system for identification of dangerous areas in the urban area targeted at the people with visual dysfunction. The idea of the system is presented comprising the attempt of application of the wireless data transmission: IR - infra-red; BLUETOOTH; RFID. The consideration comprises also the identification of the object (human), identification of the movement direction (double barrier), wireless data transmission with data on the given dangerous area, excitation of the vibration information and the feedback of the system. Also the subject of optimal installation on the urban objects was discussed. The article is a realisation of the research task in the project called "The development of the method for marking of the dangerous areas and especially important places in urban area for visually impaired people with use of the wave-vibration markers".
\end{abstract}

PACS: 41.20.--q, 46.40.-f

\section{Introduction}

The marking of the dangerous areas for people with visual dysfunction is extremely important for the safe and free commutations. Currently used methods for marking - sound signalling, shaped pavement etc. are sufficient in the stationary objects such as pedestrian crossing, staircases or rail platforms. The problem is in marking of the object that appears in certain place for a short time, for example a road work or an excavation. Also with the technological development it is possible to improve quality of life for the visually impaired people. An attempt was made to develop a system that, on one hand, would fully inform a person with a visual dysfunction about nearby danger, and, on the other hand, would not be burdensome and would not absorb too much of the user's attention. The choice was made to utilise systems for wireless data transmission as well as vibration signals to transmit information. Main guidelines are:

- development of a comfortable mechanism for delivering an information about a dangerous area to a blind person,

- development of a broadcasting-receiving mechanism coupled with a marking of a dangerous zone,

- a possibility to generate and implementation of algorithms containing information about an area,

- a necessity to detect and react to errors during data transmission,

— a possibility of self-diagnostics.

* corresponding author; e-mail: wiciak@agh.edu.pl

\section{Overview of the current solutions}

The marking of the dangerous areas for people with visual dysfunction is not a new issue. In Poland and abroad we encounter the solutions which could be classified into two groups:

- active,

- passive.

Each of the users of the public area is familiar with the acoustic signal systems. The purpose of the signalling system is the assistance for the visually impaired people at the pedestrian crossings, usually by means of sound signals or verbal signals (rare). The solution informs the commuters about the currently displayed signal. The presence of the acoustic signalling system does not guarantee the success. The mistakes in the signalling system location or in the sound level might be a reason for problems for the visually impaired person and put them at the risk. Such example might be a large number of signalling devices in one place (because of the number of crossings) on the big intersection, which combined with traffic noise presents unclear information. In every city and even on every intersection in one city there exist different kinds of acoustic signals and are not standardised. Below acoustic signalling devices are presented (Fig. 1).

Another problem is also a noise (by informational signal) which disturbs the inhabitants of the area during the night $[1,2]$. Lately some work has been undertaken on the adaptive systems.

It is is the fact also that there is not enough of the signalling devices. Also the question arises about the places without the traffic lights and thus without the possibility of installation of the acoustic signalling devices. Here the visually impaired person should rely on their own or 


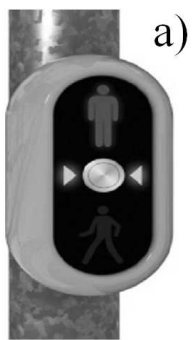

b)

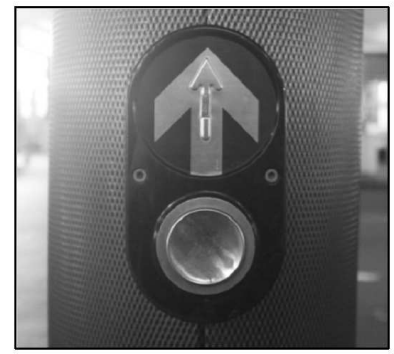

Fig. 1. Signalling device: (a) acoustic, (b) acoustic vibration.

on other people that would help to cross the street. An answer to that might be often seen (especially abroad) solution comprising specially shaped surfaces with little bumps for marking of the crossings. The simple and effective solution are effective as long as the snow does not cover them. Figure 2 presents examples of the shaped surface.
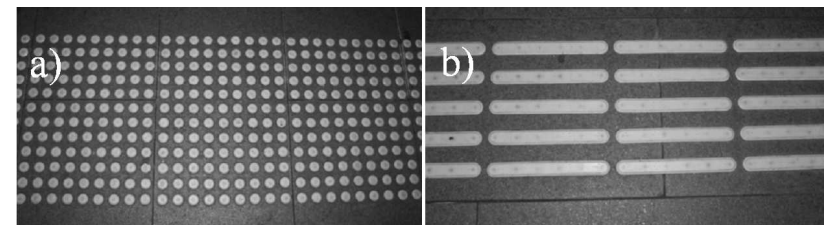

Fig. 2. Shaped surface: (a) round bumps, (b) bumpy stripes.

The people with visual dysfunction might also use the modern technological solutions, for example:

- Ultra Cane from Soundforesight (use of echolocation) allows visually impaired people to easier move around and locate the obstacles. Thanks to application of echolocation as a special module that fits a regular white cane a visually impaired person is able to recognise the area within few meters in front of them [3].

- The intelligent Blind Person Assistant (Gdańsk, Łódź), the project uses new technologies such as GSM, GPS, AUDIO and VIDEO for informing of the visually impaired person about the surrounding area [4].

— The devices "The vOICe" and "K-Sonar" $[5,6]$ use the echolocation for informing about the obstacles. The system is also integrated with the cane.

There are also some other works undertaken, e.g. identification and classification of the sources of sounds and vibrations in form of "library of acoustic events" created for teaching of the blind people of spatial orientation [7] or the concept of application of acoustic signature of the selected types of trams in spatial orientation of visually impaired people [8]. Authors claim that there exists the possibility of identification of tram type based only on the acoustic signals. According to them the assignment of the specific type of the tram to the selected route allows improvement of spatial orientation of visually impaired people in the urban area. But what if that tram is assigned to another route?

There is a number of publications $[9,10]$ in which authors disprove a common belief that "the blind people sense of smell is more keen than that of people without visual dysfunction", or "the blind people's hearing is more keen than that of people without visual dysfunction". In those works an ability of human brain named "neuroplasticity" has been proven. In case of losing one of the five senses it allows the rest of them to cooperate in such a manner that the loss of one is compensated by increasing the sensitivity of the other four. However, it is a misunderstanding that in effect of losing one of the senses the sensitivity of the rest will increase to such a degree that will provide a substantial advantage over a person without that kind of dysfunction. The same applies to the ability to memorise. Though it is true that the people with visual dysfunction demonstrate better ability to memorise, it is only so because of more training. It means that in comparison to the rest of the people, the blind people have a tendency to memorise more according to some procedures. For example, they have to find out how to get from place $A$ to place $B$ safely, while most of us does not pay attention to our surroundings during a trip. The results of the works mentioned are reflected in the research on sensory skills of the blind [10]. The researchers find some differences, however those differences are not big enough to indicate over-the-average abilities resulting from development of other senses as a result, in this particular case, of the loss of vision.

All over the country there are works pending within the project "Samorząd równych szans" ("Self-government of equal opportunities"), that will create conditions for permanent integration of the disabled people. The project consists of:

- the public space area, meaning the adaptation of public buildings for the disabled people,

- the public transport area, with a goal to adapt public transport system for the needs of the disabled people.

Above only few solutions were presented. Those solutions are not fault free and some of them even seem to be on the border of the fantasy. By analysis of those solution another (competitive) idea emerged for using of wave-vibration signalling for informing about the dangerous areas.

\section{The concept of the system}

The aim of the project is the development of the technology for identification of the dangerous areas and development of the method for teaching of the visually impaired people (children and teenagers) of identification of 
dangerous areas in the urban area based on the system of wave-vibration markers. In the beginning it was decided to focus on the dangerous areas such as:

- staircases with differentiation of direction,

— rail platform,

- pedestrian crossing,

- temporary obstacles: excavations, road works, temporary bridges.

The requirements for the system:

- definition of the general location (first signal when approaching the dangerous area),

- precise definition of the location, much higher accuracy for position of the person measurement needed (second signal about the dangerous area),

— informing the user by means of vibration signal,

- low cost,

- low power consumption.

Figure 3 presents general assumptions of the system.

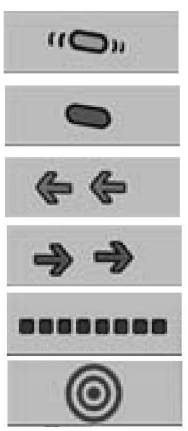

Vibrating bracelet
Actuator
Excitation signal
Warning signal
Dangerous area
Dangerous area
sensor

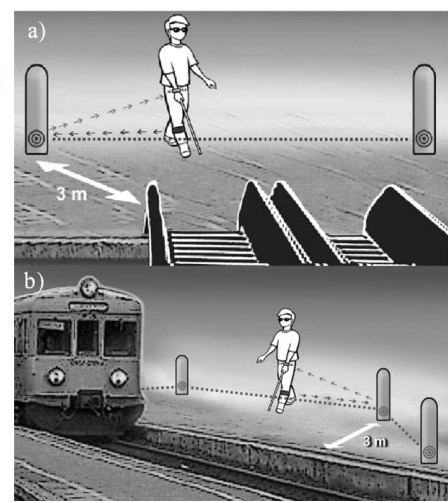

Fig. 3. Dangerous area: (a) stairs, (b) dangerous area: platform.

The realisation of the project is to ensure the creation of the transmission system (situated in the region of the dangerous area) and the reception system in form of a bracelet for the user. The transmission system will be placed on the whole surface of the dangerous area and the reception system is an individual identifier for each user and it is necessary in case when in the area two or more users of the system show up. The action of the system comprises in the fact that when the person shows up in the dangerous area the system is excited (the bracelet sends the control signal), after excitation the transmission module sends encoded signal to the bracelet, the signal with encoded information about the type of the danger. The vibrators (similar to cellular phone vibrators) in the bracelet are excited in predefined order to transmit the information to the wrist. Therefore it is important that the bracelets are individualised for the users since when two users cross the dangerous area they should not receive the contradictory information. The idea of the system includes doubling of the dangerous areas for sending general information in the area I and precise information in the area II. The emphasis is put on the functionality and ease of implementation of the solution in possibly any dangerous area, so that the area is marked permanently (e.g. under the pavement) or with possibility of moving (e.g. in form of a mat).

\section{The undertaken research}

When taking the research it was considered to use the existing solutions used in the spatial localisation and in the wireless data transmission. The solution based on the GPS technology allows only general positioning and relatively large error in the urban area (up to $10 \mathrm{~m}$ ). Also the application of the satellite technology is not possible to use in the buildings or underground. Another solution that would provide data transmission and spatial positioning is infra-red data association (IrDA). The solution is characterised by simple and inexpensive implementation, low power consumption, efficient and reliable data transfer. Unfortunately it has one major disadvantage that disqualifies the IrDA technology in the presented system - it requires direct visibility between the sender and the receiver.

That is why another technology was considered a wireless short range transmission system - BLUETOOH. One of its advantages is a range defined by the power class:

- class 1 (100 mW) longest range, up to $100 \mathrm{~m}$,

— class $2(2.5 \mathrm{~mW})$ most common, range up to $10 \mathrm{~m}$,

- class $3(1 \mathrm{~mW})$ rarely used, range up to $1 \mathrm{~m}$,

another is relatively cheap implementation. Its disadvantage is the connection time of the devices and the problem with measuring of the precise position without building sophisticated positioning system.

Finally a decision was made to try radio frequency identification (RFID) systems, which is widely used technology for localisation of human and goods. Figure 4 presents the concept of the RFID system.

It was decided to build the first prototype stand with use of RFID technology to define its actual usability in the building of the whole system.

In terms of work frequencies used three types of "tags" can be identified:

- close field tags — with work frequency range $10-500 \mathrm{kHz}$ and $10-15 \mathrm{MHz}$;

- far field tags — with work frequency range 860-960 MHz; 


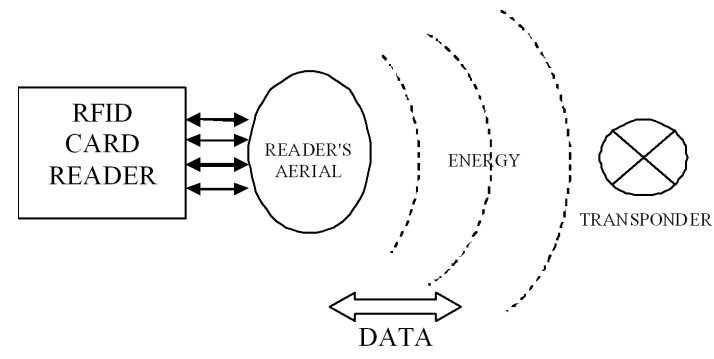

Fig. 4. Schema of RFID system.

- tags working in frequency range $2.4-5.0 \mathrm{GHz}(\mathrm{mi}-$ crowave).

The project uses passive tags (frequency range up to $100 \mathrm{MHz}$ ), which use magnetic induction. It works this way: electric current in receiver antenna creates a magnetic field. When the tag enters the field, an electric current is induced in the tag. This delivers the energy to send information contained in tag. Summing up: tag's contain information that is send due to magnetic field changes.

The range of close and far fields is expressed by equation

$$
L=\frac{c}{2 \pi f},
$$

where $L$ - range of field $[\mathrm{m}], c$ - velocity of light $[\mathrm{m} / \mathrm{s}]$, $f$ - wave frequency $[\mathrm{Hz}]$.

Further studies will address subjects like: range of field, work effectiveness/efficiency and the alignment of antenna.

\section{Conclusion}

It is the fact that there exist many projects and readily available solutions that might help people with visual dysfunction in the commutation but they have not been consulted or tested with their users and - despite the good intentions of their authors - their effect is opposite to the expected.

Example might be before mentioned problems with wrong positioning of the acoustic signalling devices, or simply lack of them. A significant problem is the lack of functionality of the most sophisticated systems but absorbing too much attention of the visually impaired person, or being difficult to learn. That means the subject is still interesting and worth consideration.

The aim of the project is building of the system that is easy in operation, not absorbing too much attention of the user and giving clear information about the dangerous area. The system should be characterised by low cost, easy implementation in different places (indoors and outdoors) and low power consumption - the limit is set

for 5 day without recharging. It is an interesting fact that the system is in the initial stage of development and is already interesting for the groups of visually impaired people, which is presented in Fig. 5 [11].
a) increase of skills....
b) ability to use......
c) use of equipment.....
d) improvement of walking techniques

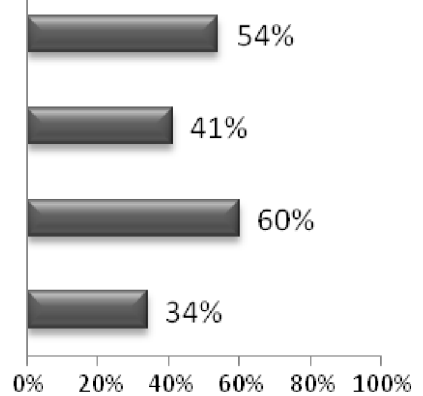

Fig. 5. What would influence the improvement of my skills in commutation? [11].

During next stages of the works, tests concerning usefulness of the RFID in the project will be carried out, and a prototype research stand utilising aforementioned technologies will be constructed. Moreover, a simultaneous research on an alternative solution that would fulfill requirements of the project will be conducted.

\section{Acknowledgments}

The work was financed by the development project NR17001706 founded by the National Centre of Research and Development (NCBiR).

\section{References}

[1] H. Lubawy, TYFLOŚWIAT No. 1, 12 (2008).

[2] http://czt.zm.org.pl/?a=sygnalizacja-08a .

[3] http://www.altix.pl .

[4] Research Project MNiI nr 3T11D03129.

[5] H. Lubawy, TYFLOŚWIAT No. 1, 16 (2009).

[6] http://www.batforblind.co.nz/.

[7] E. Skrodzka, F. Tomaszewski, B. Czechyra, in: 56 Open Seminar on Acoustic, Goniadz nad Biebrza 1518.09.2009, p. 511.

[8] E. Skrodzka, F. Tomaszewski, B. Czechyra, in: 56 Open Seminar on Acoustic, Goniadz nad Biebrza 1518.09.2009, p. 415.

[9] N. Lessard, M. Paré, F. Lepore, M. Lassonde, Nature 395, 278 (1998).

[10] H.-H. Lai, Y.-C. Chen, Int. J. Industr. Ergonom. 36, 565 (2006).

[11] J. Wiciak, in: The 20th Int. Congress on Acoustics, Sydney (Australia), Eds. M. Burgess, J. Davey, C. Don, T. McMinn, Australian Acoustical Society 2010, p. 23. 